\title{
Ultraperipheral production of very small number of particles in ultrarelativistic heavy ion collisions *
}

\author{
Antoni Szczurek ${ }^{1,2, a}$ and Mariola Kłusek-Gawenda ${ }^{1}$ \\ ${ }^{1}$ The Henryk Niewodniczański Institute of Nuclear Physics PAN, PL-31-342 Kraków \\ ${ }^{2}$ University of Rzeszów, PL-35-959 Rzeszów
}

\begin{abstract}
We review our recent results on exclusive production of $\mu^{+} \mu^{-}$, heavy quark antiquark and meson-antimeson pairs in ultraperipheral, ultrarelativistic heavy ion collisions.
\end{abstract}

\section{Introduction}

Ultrarelativistic collisions of heavy ions provide a nice oportunity to study $\gamma \gamma$ collisions. An enhancement of the cross section for the reactions of this type compared to proton-proton or $\mathrm{e}^{+} \mathrm{e}^{-}$collisions which is caused by large charges of the colliding ions is expected. In this type of reactions virtual (almost real) photons couple to the nucleus as a whole. Naively the enhancement of the cross section is proportional to $Z_{1}^{2} Z_{2}^{2}$ which is a huge factor. We have discussed that the inclusion of realistic charge distributions and realistic nucleus charge form factor reflects in a cross section smaller than those obtained by other models using naive predictions, usually found in literature.

\section{Equivalent Photon Approximation}

The Equivalent Photon Approximation [1] is a standard semi-classical alternative to the Feynman rules for calculating cross sections of electromagnetic interactions. The method is based on the observation that the electric and magnetic fields of a fast-moving charged particle are nearly transverse to the direction of motion (see figure 1). The total cross section is calculated by the convolution:

$\sigma\left(A A \rightarrow A A X_{1} X_{2} ; s_{A A}\right)=\int \hat{\sigma}\left(\gamma \gamma \rightarrow X_{1} X_{2} ; W_{\gamma \gamma}\right) S_{a b s}^{2}(\mathbf{b}) N\left(\omega_{1}, \mathbf{b}_{1}\right) N\left(\omega_{2}, \mathbf{b}_{2}\right) \frac{W_{\gamma \gamma}}{2} d^{2} \mathbf{b}_{1} d^{2} \mathbf{b}_{2} d W_{\gamma \gamma} d Y_{X_{1} X_{2}}$,

where $X_{1}$ and $X_{2}$ are leptons, quarks or mesons, $S_{a b s}^{2}(\mathbf{b})=\theta\left(\left|\mathbf{b}_{1}-\mathbf{b}_{2}\right|-2 R_{A}\right)$ is the absorption factor (ultraperipheral collisions), $N(\omega, \mathbf{b})$ the photon flux, $\omega$ is the energy of photon and $Y_{X_{1} X_{2}}=\frac{1}{2}\left(y_{X_{1}}+y_{X_{2}}\right)$ rapidity of the outgoing $X_{1} X_{2}$ system.

\footnotetext{
${ }^{\star}$ This work is supported by the Polish grant N DEC-2011/01/B/ST2/04535

a e-mail: Antoni.Szczurek@ifj.edu.pl
} 

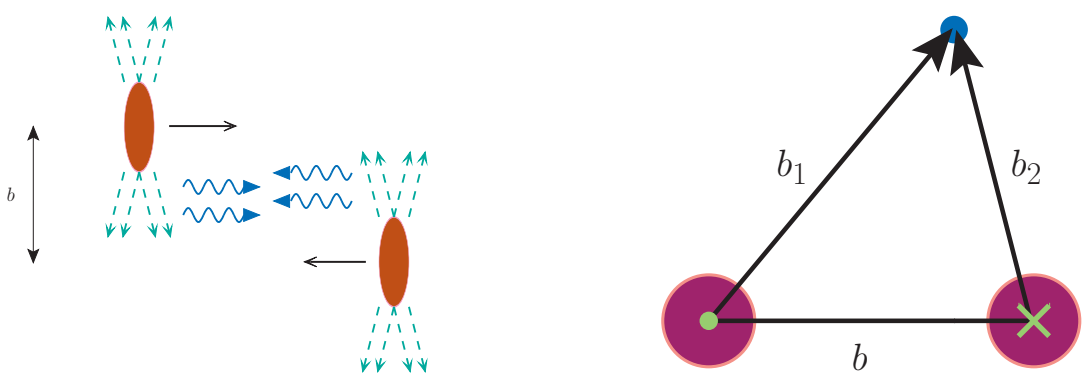

Figure 1. Left panel - The Weizsäcker-Williams method. Right panel - The quantities used in the impact parameter calculations.

\section{Exclusive production of $\mu^{+} \mu^{-}$pairs}

Elementary cross section for charged leptons can be calculated within Quantum Electrodynamics. In Ref. [2] we have presented several distributions for exclusive muon-pair production in nucleusnucleus collisions. Realistic (Fourier transform of charged density) charge form factors of nuclei are used and the corresponding results are compared with the cross sections calculated with monopole form factor often used in the literature. It was discussed in the literature that higher-order are not important for the presented distributions [3].
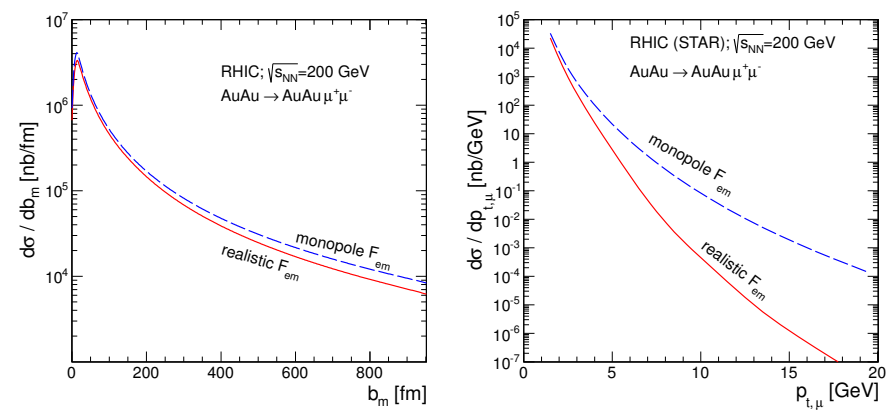

Figure 2. Left panel - The cross section as a function of impact parameter. Right panel - Muon transverse momentum distribution for STAR conditions: $y_{\mu^{ \pm}} \in(-1,1), p_{t, \mu^{ \pm}} \geqslant 1 \mathrm{GeV}$.

\section{Exclusive production of $c \bar{c}$ and $b \bar{b}$}

We have calculated also cross sections for exclusive production of charm-anticharm and bottomantibottom pairs, for the $Q \bar{Q} g$ and $Q \bar{Q} q \bar{q}$ final state, as well as for the single-resolved components in the high-energy peripheral lead-lead collisions for the LHC energy $\sqrt{s_{N N}}=5.5 \mathrm{TeV}$. In figure 3 we compare the contributions of different mechanisms discussed in Ref. [4]. Large cross sections have been found in the case of charm quarks production. In table 1 we show partial contribution of different subprocesses discussed in our paper. 

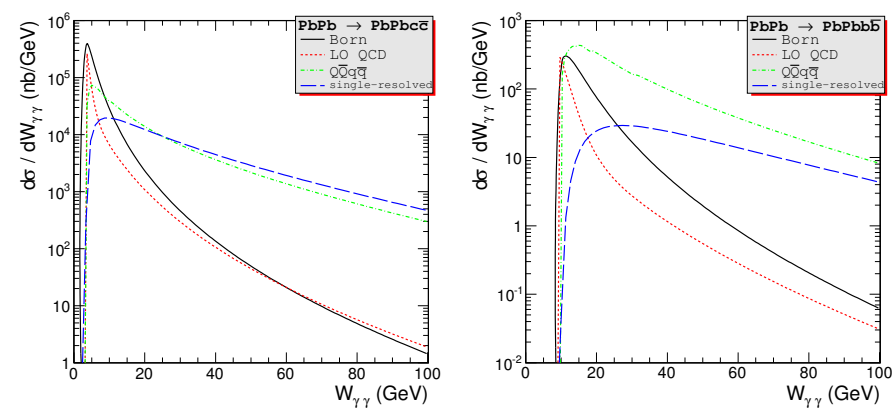

Figure 3. Nuclear cross section as a function of $\gamma \gamma$ subsystem energy $W_{\gamma \gamma}$ for the $P b P b \rightarrow P b P b c \bar{c}$ (left panel) and for the $P b P b \rightarrow P b P b b \bar{b}$ (right panel).

Table 1. Partial contributions of different mechanisms at $\sqrt{s_{N N}}=5.5 \mathrm{TeV}$.

\begin{tabular}{cccccc}
\hline & $\sigma_{\text {tot }}$ & Born & QCD corrections & $Q \bar{Q} q \bar{q}$ & Single-resolved \\
\hline$c \bar{c}$ & $2.47 \mathrm{mb}$ & $42.5 \%$ & $14.6 \%$ & $27.1 \%$ & $15.8 \%$ \\
$b \bar{b}$ & $10.83 \mu \mathrm{b}$ & $18.9 \%$ & $7.7 \%$ & $64.5 \%$ & $8.9 \%$ \\
\hline
\end{tabular}

\section{Double $J / \Psi$ production}

In Ref. [5] we have calculated the cross section for the $\gamma \gamma \rightarrow J / \Psi J / \Psi$ process. Two mechanisms are considered: box (two-loop) diagrams (dashed line) of the order of $\mathrm{O}\left(\alpha_{e m}^{2} \alpha_{s}^{2}\right)$ and two-gluon exchange (dotted line) of the order of $\mathrm{O}\left(\alpha_{e m}^{2} \alpha_{s}^{4}\right)$. The first mechanism is calculated in the heavy-quark nonrelativistic approximation while in the second case we also include the effects of quantum motion of quarks in the bound state.
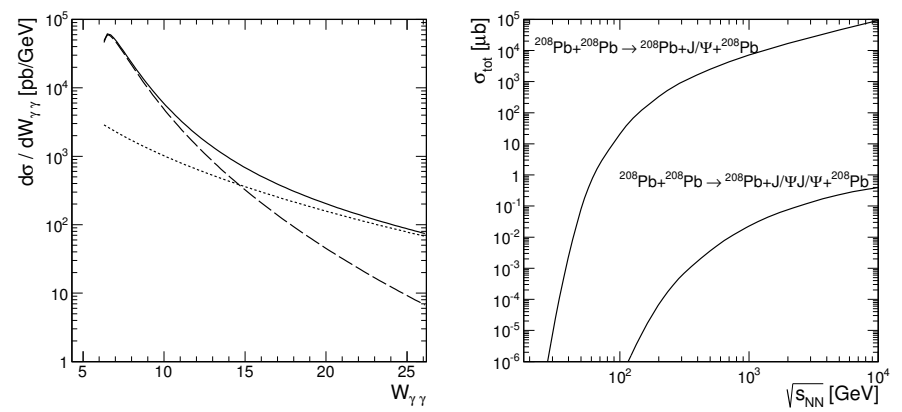

Figure 4. Left panel - Invariant mass of $J / \Psi J / \Psi$ pair distribution in ${ }^{208} \mathrm{~Pb}+{ }^{208} \mathrm{~Pb} \rightarrow{ }^{208} \mathrm{~Pb}{ }^{208} \mathrm{~Pb} J / \Psi J / \Psi$ process for $\sqrt{s_{N N}}=2.76 \mathrm{TeV}$. Right panel - Cross section as a function of initial energy for the $\mathrm{PbPb} \rightarrow \mathrm{PbPb} / \Psi J / \Psi$ process and for the $\mathrm{PbPb} \rightarrow \mathrm{PbPb} J / \Psi$ process. 


\section{$6 \rho^{0} \rho^{0}$ and $\pi \pi$ pair production}

In Ref. [6-8] we have calculated, for the first time, differential distributions for two $\rho^{0}$ mesons and for pion-pion production in exclusive, ultraperipheral, ultrarelativistic collisions. In figure 5 we show theoretical distribution for the full phase space (upper solid lines) and with extra cuts on $z=\cos \theta$. At lower energies the result does not depend on the angulat cuts. the big differences start in the region where the elementary cross section can be understood in terms of the BL pQCD and handbag mechanisms. The peaks reflect complicated energy dependence of the elementary $\gamma \gamma \rightarrow \pi \pi$ cross section [8].
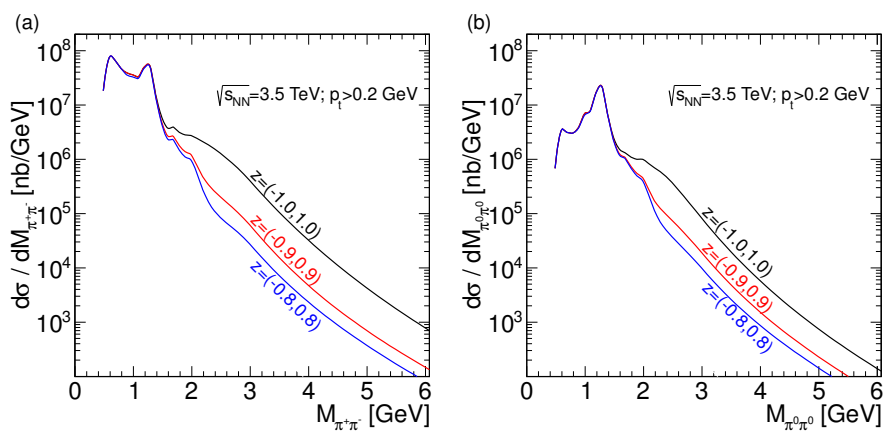

Figure 5. Total cross section for $\gamma \gamma \rightarrow \pi^{+} \pi^{-}$(left panel) and $\gamma \gamma \rightarrow \pi^{0} \pi^{0}$ (right panel) as a function of the dipion mass at the LHC energy $\sqrt{s_{N N}}=3.5 \mathrm{TeV}$ and $p_{t, \pi}>0.2 \mathrm{GeV}$.

\section{Conclusions}

We have presented some examples of processes that are or could be studied at RHIC or LHC. In all considered cases we have obtained measurable cross sections. We have pointed out that the inclusion of realistic charge form factor is necessary to obtain realistic cross sections and particle distributions for nuclear collisions.

\section{References}

[1] J.D. Jackson, Classical Electrodynamics 2nd ed. (Wiley, New York, 1975) p. 722.

[2] M. Kłusek-Gawenda and A. Szczurek, Phys. Rev. C82 014904 (2010).

[3] U.D. Jentschura and V.G. Serbo, Eur. Phys. J. C64 309 (2009).

[4] M. Kłusek-Gawenda, A. Szczurek, M. V. T. Machado, and V. G. Serbo, Phys. Rev. C83 024903 (2011).

[5] S. Baranov, A. Cisek, M. Kłusek-Gawenda, W. Schäfer and A. Szczurek, Eur. Phys. J. C73 2335 (2013).

[6] M. Kłusek, A. Szczurek, and W. Schäfer, Phys. Lett. B674 92 (2009).

[7] M. Kłusek-Gawenda and A. Szczurek, Phys. Lett. B700 322 (2011).

[8] M. Kłusek-Gawenda and A. Szczurek, Phys. Rev. C87 054908 (2013). 